This item was submitted to Loughborough's Research Repository by the author.

Items in Figshare are protected by copyright, with all rights reserved, unless otherwise indicated.

\title{
Numerical predictions of turbulent underexpanded sonic jets using a pressure-based methodology
}

PLEASE CITE THE PUBLISHED VERSION

PUBLISHER

Professional Engineering Publishing / @ IMECHE

VERSION

VoR (Version of Record)

LICENCE

CC BY-NC-ND 4.0

\section{REPOSITORY RECORD}

Birkby, Paul, and Gary J. Page. 2009. "Numerical Predictions of Turbulent Underexpanded Sonic Jets Using a Pressure-based Methodology". figshare. https://hdl.handle.net/2134/5052. 
This item was submitted to Loughborough's Institutional Repository (https://dspace.lboro.ac.uk/) by the author and is made available under the following Creative Commons Licence conditions.

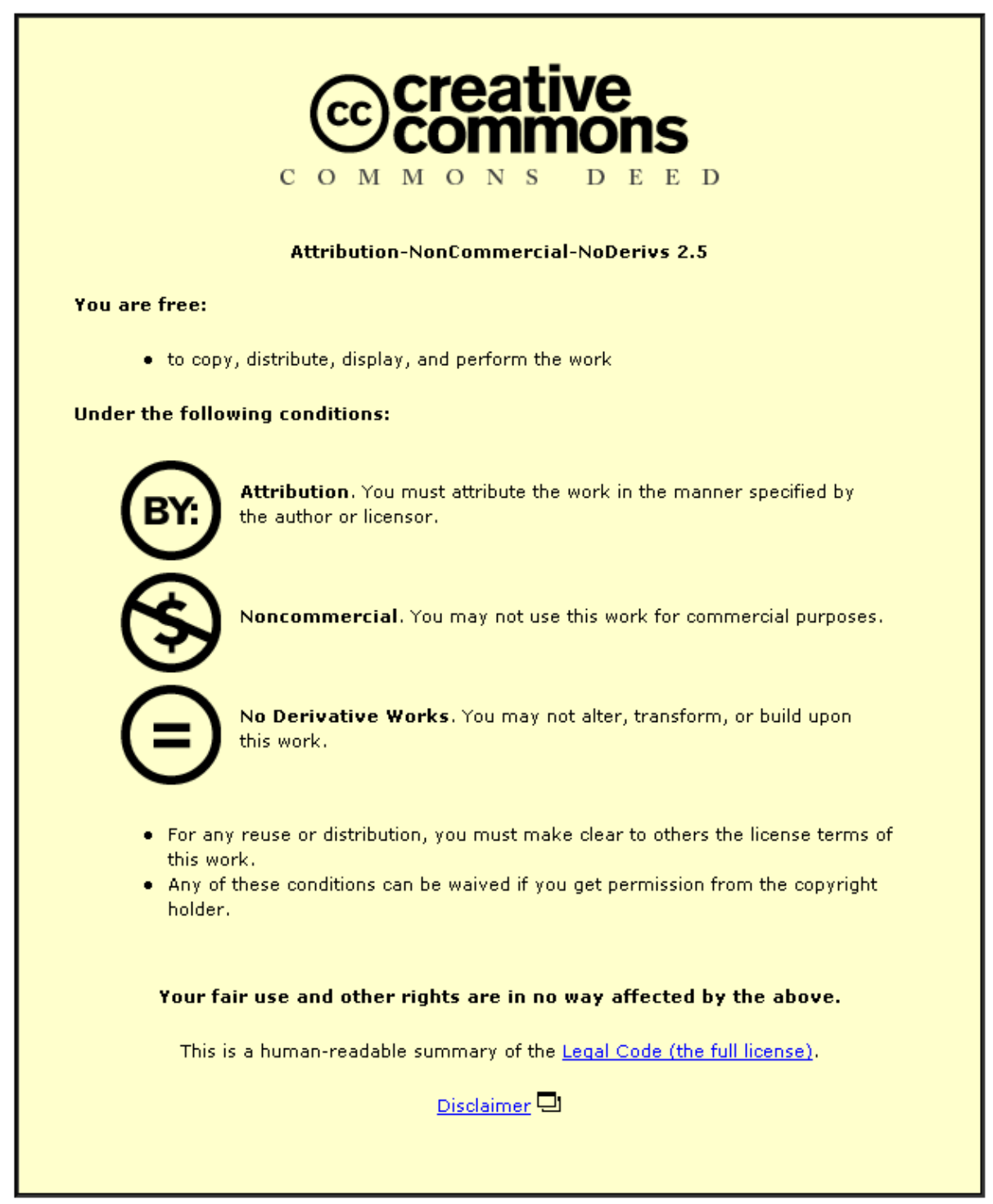

For the full text of this licence, please go to: http://creativecommons.org/licenses/by-nc-nd/2.5/ 


\title{
Numerical predictions of turbulent underexpanded sonic jets using a pressure-based methodology
}

\author{
P Birkby ${ }^{1}$ and G J Page ${ }^{2 *}$ \\ ${ }^{1}$ Department of Engineering, University of Cambridge, UK \\ ${ }^{2}$ Department of Aeronautical and Automotive Engineering, Loughborough University, Leicestershire, UK
}

\begin{abstract}
The objective of this work is to model underexpanded turbulent sonic jets. A pressure-based computational fluid dynamics methodology has been employed, incorporating extensions to handle high speed flows. A standard two-equation turbulence model is used, with an optional compressibility correction. Comparison with experimental jet centre-line Mach number showed the correct shock cell wavelength but a too rapid decay. The compressibility correction had no effect on the shock cell decay but increased the potential core length to give better agreement with experiment. Calculations for nozzle pressure ratios up to 30 showed the variation of Mach disc location in good agreement with experiment. For nozzle pressure ratios above 6, unsteady solutions were observed, emanating from the intersection of the Mach disc with the shear layer. Experimental work has identified similar large-scale instabilities; the peak mode of the prediction had a Strouhal number of 0.16 , close to experimental values.
\end{abstract}

Keywords: jet, underexpanded, turbulent flow, computational fluid dynamics (CFD)

\section{NOTATION}

$a \quad$ speed of sound
$C_{\mu}, C_{\varepsilon 1}, C_{\varepsilon 2}$

turbulence model constants

$d, r \quad$ jet nozzle diameter, radius

$H \quad$ total enthalpy

$l \quad$ first shock cell length

$k \quad$ turbulence kinetic energy

$M_{\tau} \quad$ turbulence Mach numbers

NPR nozzle pressure ratio

$P \quad$ pressure

$P_{k} \quad$ production rate of $k$

$u, v \quad$ Cartesian velocity components

$v_{j} \quad$ velocity vector

$\varepsilon \quad$ dissipation rate of $k$

$\gamma \quad$ ratio of specific heats

$\mu \quad$ viscosity

$\mu_{\mathrm{t}} \quad$ turbulent viscosity

$\rho \quad$ density

$\sigma_{k}, \sigma_{\varepsilon} \quad$ turbulence model constant

$\tau_{i j} \quad$ shear stress tensor

\author{
Superscripts \\ - $\quad$ conventional ensemble average \\ density-weighted ensemble average \\ " density-weighted fluctuation
}

\section{INTRODUCTION}

High-speed jet flows are of significant interest in many fields of engineering. These include propulsive jets in aerospace applications and the accidental release of highpressure combustible gases into the atmosphere. The present work concentrates on underexpanded jets with sonic velocities expanding into nominally quiescent conditions. This relates to convergent nozzles in propulsive applications or the release of high pressure gases through an aperture.

The structure of underexpanded jets has been well documented [1, 2]. They contain a complex periodic shock cell structure created by the imbalance of static pressure between the jet core and ambient. If the nozzle pressure ratio (NPR) is defined as the ratio of jet exit total pressure to ambient static pressure, then for nozzle pressure ratios of around 4 the jet becomes highly underexpanded and a normal shock or Mach disc is formed. Downstream of the Mach disc a pocket of subsonic flow will be created in the jet core. Experimental measurements of underexpanded jets are relatively limited owing to the difficulties arising from varying static pressures, high velocities and shock waves.
The MS was received on 26 November 2000 and was accepted after revision for publication on 5 March 2001.

* Corresponding author: Department of Aeronautical and Automotive Engineering, LoughboroughUniversity, Leicestershire LE11 3TU, UK. 
Early work concentrated on global quantities such as shock cell wavelength and Mach disc location $[3,4]$. Measurements of the flow properties of underexpanded jets were initially limited to pressure probe measurements $[\mathbf{5}, \mathbf{6}]$ or else the measurements were of the far field flow only [7]. More recently laser Doppler anemometry and laser-induced fluorescence have been used [8], although the measurements were limited to moderately underexpanded jets. Reliable measurements of velocity and turbulence quantities are not at present available but are clearly important for the validation of modelling techniques and for jets undergoing combustion.

Computational fluid dynamics (CFD) modelling of moderately underexpanded jets has been reported in several studies. The earliest work [9] used the Euler equations with varying success. Several studies have employed a parabolized Navier-Stokes method $[\mathbf{8}, \mathbf{1 0}, \mathbf{1 1}]$. The method was found to overpredict the shock structure and to underpredict the mixing rate of sonic jets. Use of a Navier-Stokes solver along with the two-equation $k-\varepsilon$ model showed promising results downstream of the shockcontaining region [12]. Highly underexpanded jets have been modelled much less. Both the Euler and the NavierStokes equations were used to model the first five diameters of a jet with a nozzle pressure ratio of 6.76 [13]. Reference [9] reports comparisons of the computed Mach disc locations with experiments [3], although no other measurement of these jets was reported. The earlier work of reference [12] was extended to compute highly underexpanded jets [14]; the predictions were found to be in good agreement with experimental observations of Mach disc location, but the emphasis of the work was on the mean velocity and mixture fractions downstream.

One recurring issue in the modelling of underexpanded jets using the Navier-Stokes equations is the effect of compressibility on turbulence. Experimental work [15] demonstrated that as the convective Mach number increased then the growth rate of a turbulent shear layer decreased, an effect that is not reproduced by standard turbulence models. Convective Mach number is defined as the ratio of the mean velocity difference across the layer to the sum of the sound speeds in the two streams. Several works have therefore considered compressibility corrections. Experimental data were used in an empirical manner to attenuate the turbulent viscosity as the convective Mach number increased [8]. Reference [11] applied a model based on two length scales obtained from the $k-\varepsilon$ model and experimental data. Modelling sonic jets with a parabolized Navier-Stokes model and a compressibility correction was found to cause a deterioration in the computed accuracy since the mixing was already underpredicted. This was not the case for supersonic jets or with a Navier-Stokes solver [12, 14].

Early work [16] introduced the concept of dilatation dissipation (in addition to solenoidal dissipation) for high Reynolds number compressible turbulence. This was predicated on the existence of shock-like structures within energetic eddies. By modelling the dilatation dissipation as a function of turbulence Mach number and solenoidal dissipation, the correct reduction in shear layer spreading rate with convective Mach number was reproduced. Reference [17] proposed a similar modelling approach based on direct numerical simulations of compressible, isotropic turbulence. The model was implemented in a second-order closure model [18] where it was found to reproduce the dependence of mixing layer growth on the convective Mach number. It has also been implemented in a two-equation model applied to underexpanded jets [12], where it not only improved downstream mixing results but had an extremely favourable effect on the shock resolution.

More recently $[\mathbf{1 9}, \mathbf{2 0}]$, there has been considerable evidence that the influence of dilatation dissipation and pressure dilatation is small in a compressible mixing layer (even when eddy shocklets are observed) and that support for the initial theory came from simulations of isotropic or fully homogeneous compressible turbulence. It was shown that the reduction in growth rate was due to reduced pressure fluctuations leading to a reduction in the pressure-strain term [20] and is related to the anistropy of the Reynolds stress tensor [19]. Although the models based on compressible dissipation are physically incorrect, they nevertheless give a reasonable reproduction of the spreading rate reduction with convective Mach number.

The objectives of the current work are to model highly underexpanded jets exhausting into a quiescent atmosphere, in order to predict the occurrence and location of the Mach disc and the turbulent mixing in the shear layer. In the following section the CFD methodology is described in detail. Following this, the results obtained for underexpanded sonic jets at various nozzle pressure ratios are discussed along with the application of a compressibility correction to the turbulence model and some preliminary observations regarding jet flow unsteadiness.

\section{METHODOLOGY}

The methodology employed in the current work solves the time-dependent, density-weighted ensemble-averaged conservation equations for mass and momentum, together with the assumption of constant total enthalpy:

$$
\begin{aligned}
& \frac{\partial}{\partial t} \bar{\rho}+\frac{\partial}{\partial x_{j}} \bar{\rho} \tilde{v}_{j}=0 \\
& \frac{\partial}{\partial t} \bar{\rho} \tilde{v}_{j}+\frac{\partial}{\partial x_{j}}\left[\bar{\rho} \tilde{v}_{i} \tilde{v}_{j}+\bar{P} \delta_{i j}-\left(\tau_{i j}-\bar{\rho} \widetilde{\left.v_{i}^{\prime \prime}\right)_{j}^{\prime \prime}}\right)\right]=0 \\
& H=\frac{\gamma}{\gamma-1} \frac{\bar{P}}{\bar{\rho}}+\frac{1}{2} \tilde{v}_{i} \tilde{v}_{j}
\end{aligned}
$$

The mass-weighted Reynolds stress tensor is modelled using the Boussinesq assumption, 


$$
\begin{aligned}
\bar{\tau}_{i j}-\bar{\rho} \widetilde{v_{i}^{\prime \prime} v_{j}^{\prime \prime}}= & \left(\mu+\mu_{\mathrm{t}}\right)\left(\frac{\partial \tilde{v}_{i}}{\partial x_{j}}+\frac{\partial \tilde{v}_{j}}{\partial x_{i}}\right) \\
& -\frac{2}{3} \delta_{i j}\left[\left(\mu+\mu_{\mathrm{t}}\right) \frac{\partial \tilde{v}_{l}}{\partial x_{l}}+\bar{\rho} \tilde{k}\right]
\end{aligned}
$$

and the turbulent viscosity is found from a mass-weighted variant of the standard high Reynolds number $k-\varepsilon$ model [21], with the standard set of constants:

$$
\begin{aligned}
& \frac{\partial}{\partial t} \bar{\rho} \tilde{k}+\frac{\partial}{\partial x_{j}}\left(\bar{\rho} \tilde{k} \tilde{v}_{j}-\frac{\mu_{\mathrm{t}}}{\sigma_{k}} \frac{\partial}{\partial x_{j}} \tilde{k}\right)=P_{k}-\bar{\rho} \tilde{\varepsilon} \\
& \frac{\partial}{\partial t} \bar{\rho} \tilde{\varepsilon}+\frac{\partial}{\partial x_{j}}\left(\bar{\rho} \tilde{\varepsilon} \tilde{v}_{j}-\frac{\mu_{\mathrm{t}}}{\sigma_{k}} \frac{\partial}{\partial x_{j}} \tilde{\varepsilon}\right)=\frac{\tilde{\varepsilon}}{\tilde{k}}\left(C_{\varepsilon 1} P_{k}-C_{\varepsilon 2} \bar{\rho} \tilde{\varepsilon}\right) \\
& \mu_{\mathrm{t}}=C_{\mu} \frac{\tilde{k}^{2}}{\tilde{\varepsilon}}
\end{aligned}
$$

In order to model the observed reduction in spreading rate of a high-speed shear layer, the modifications proposed in reference [17] have been implemented in the $k-\varepsilon$ turbulence model. This introduces extra terms as a function of the local turbulence Mach number,

$$
M_{\tau}=\frac{\sqrt{2 k}}{a}
$$

the addition of a sink term in the $k$ equation,

$$
S_{k}=-\bar{\rho} M_{\tau}^{2} \widetilde{\varepsilon}
$$

and a scaling of the turbulent viscosity,

$$
\mu_{\mathrm{t}}=C_{\mu} \bar{\rho} \frac{\tilde{k}^{2}}{\left(1+M_{\tau}^{2}\right) \tilde{\varepsilon}}
$$

While more recent work $[\mathbf{1 9}, \mathbf{2 0}]$ has shown that this modification based on dilatational terms is physically incorrect, it has nevertheless been shown to reproduce correctly the dependence of mixing layer growth on convective Mach number [18].

The governing equations are discretized using a finite volume technique on a Cartesian mesh with a twodimensional axisymmetric coordinate system. A staggered mesh approach is employed: the mass and turbulence model equations are integrated over the main control volume, while the control volumes for the components of momentum are displaced in the appropriate direction, such that one set of faces is positioned at the centre of the main control volume. It should be noted that the primary set of variables is defined as quantities per unit volume, rather than per unit mass; thus momentum is considered a primary variable (on which a conservation equation can be applied), rather than velocity, which must now be derived from density and momentum. As shown in reference [22], when applied to pressure-based algorithms, this has a very beneficial effect on accuracy for compressible flows, in particular those containing shock waves.

The governing equations (1), (2), (5) and (6) can be cast into the form of a model transport equation for the variable $\Phi$, where $\Phi$ corresponds to $\rho, \rho u, \rho v, \rho \varepsilon$ or $\rho k$ :

$$
\begin{aligned}
\frac{\partial \Phi}{\partial t}+ & \frac{\partial}{\partial x}\left[\Phi u-\Gamma \frac{\partial}{\partial x}\left(\frac{\Phi}{\rho}\right)\right] \\
+ & \frac{\partial}{\partial y}\left[\Phi v-\Gamma \frac{\partial}{\partial y}\left(\frac{\Phi}{\rho}\right)\right]=S_{\Phi}
\end{aligned}
$$

The source $S_{\Phi}$ contains the remaining terms which do not fit into the model equation as well as the true sources.

The numerical scheme uses a basic first-order upwind scheme which is discretized in an implicit manner; a higher-order discretization using a total variation diminishing (TVD) principle is implemented as an explicit correction to the basic upwind scheme. All diffusive terms are discretized using central differencing.

The basic upwind scheme determines the control volume face velocities using central differencing; the sign of this velocity is then used to determine which of the adjacent control volume values of $\Phi$, together with the face velocity, should be used to construct the flux at the face. For example, the basic upwind flux at the right face of cell $i$ is

$$
\Phi_{i+1 / 2} u_{i+1 / 2}=u_{i+1 / 2}^{+} \Phi_{i}+u_{i+1 / 2}^{-} \Phi_{i+1}
$$

where

$$
u_{i+1 / 2}^{ \pm}=\frac{u_{i+1 / 2} \pm\left|u_{i+1 / 2}\right|}{2}
$$

The TVD correction consists of additional higher-order terms to equation (12),

$$
\mathrm{HOT}=\frac{1}{2}\left(u_{i+1 / 2}^{+} \Delta_{i+1 / 2}^{+}-u_{i+1 / 2}^{-} \Delta_{i+1 / 2}^{-}\right)\left(\Phi_{i+1}-\Phi_{i}\right)
$$

and the limiter function is

$$
\Delta_{i+1 / 2}^{ \pm}=\max \left[0, \min \left(\beta r_{i+1 / 2}^{ \pm}, 1\right), \min \left(r_{i+1 / 2}^{ \pm}, \beta\right)\right]
$$

based on the forward and backward monitor functions:

$$
\begin{aligned}
& r_{i+1 / 2}^{+}=\frac{\Phi_{i}-\Phi_{i-1}}{\Phi_{i+1}-\Phi_{i}} \\
& r_{i+1 / 2}^{-}=\frac{\Phi_{i+2}-\Phi_{i+1}}{\Phi_{i+1}-\Phi_{i}}
\end{aligned}
$$

This scheme is second-order accurate in smooth regions 
and the limiter ensures monotonicity. The compression parameter $\beta$ steepens discontinuities; for the current work a value of unity has been employed which reproduces the 'minmod' scheme.

This discretization leads to a set of implicit scalar equations for $\Phi$ which are solved using a line Gauss-Seidel technique. The equations are coupled together and advanced in time using a pressure-based approach. Typically in many high-speed flow problems, density-based approaches are employed - however, at low Mach numbers these algorithms become inefficient and often unstable. The flows of interest here contain a high-speed jet core exhausting into ambient conditions where the flow is at rest; a pressure-based approach with a correctly formulated compressible treatment can accurately compute flows containing both high and low Mach number zones [23].

The equation for conservation of mass is converted into an implicit equation for change in pressure (or 'pressure correction') by considering the linkage between changes in density and pressure (via the equation of state) and the linkage between changes in velocity and changes in pressure gradient (via a truncated form of the discretized momentum equations). The mass conservation equation does not conform well with the model scalar convection-diffusion equation in that there are no diffusion terms. If the convected density term is discretized using central differencing then the algorithm becomes unstable when the local Mach number exceeds unity; conversely, upwind differencing of density is stable but leads to a diffusive solution with highly smeared shock waves. The current work effectively introduces an artificial diffusion term by blending the central and upwind discretizations [24]. The blending is controlled by the local Mach number; in subsonic zones the scheme essentially reproduces central differencing, while for local Mach numbers above 2.5 the scheme is predominantly upwind. This deceptively simple technique has been found to be robust and accurate. Although the alternative 'retarded pressure' approach [22] gives crisper shock capturing, it is not as robust, failing for high Mach number flows.

A backward Euler implicit method is used for the temporal discretization. For steady state predictions, a local time step is computed on the basis of a prescribed CourantFriedrichs-Lewy (CFL) number, with a typical value being between 3 and 5. Unsteady predictions use a global time step calculated a priori from an estimate of the CFL number at the jet exit. Iteration can be used within individual timesteps to converge the Euler implicit equations; however, with the size of timestep employed in these calculations, this was not found to improve convergence or temporal accuracy.

\section{RESULTS}

\subsection{Computational domain and boundary conditions}

All calculations presented in the present work have used a jet nozzle diameter of $25 \mathrm{~mm}$, resulting in a range of
Reynolds numbers from $1.0 \times 10^{6}$ to $2.3 \times 10^{6}$ (based on nozzle diameter and fully expanded conditions). The computational domain extended 40 nozzle diameters downstream from the jet exit and 15 nozzle diameters normal to the jet centre-line. The predictions presented here use 600 cells in the streamwise direction and 210 transverse to the flow. Moderate clustering was used in the jet shear layer (smallest transverse spacing of 0.01 diameters) and near the nozzle exit (smallest axial spacing of 0.01 diameters). Comparisons to show the influence of grid resolution are discussed in the following section. At the jet nozzle exit plane, the numerical boundary conditions impose a given total pressure and total temperature; the static pressure is extrapolated from the interior in order to determine the jet velocity, density and temperature. For all the cases presented here, the jet total pressure is above critical and the boundary conditions correctly reproduce a sonic jet exit velocity. For the plane adjacent to the nozzle and at the far field boundaries normal and parallel to the jet, a condition of constant static pressure is imposed; depending on the local pressure and flow direction adjacent to the boundary this will generate an inflow or outflow. The turbulence model jet inflow conditions were prescribed on the basis of a low turbulence intensity (5 per cent), a sonic reference velocity and a length scale of 5 per cent of the nozzle diameter; for the ambient inflow conditions a smaller reference velocity of approximately $10 \mathrm{~m} / \mathrm{s}$ was used. The solution was found to be relatively insensitive to the inflow turbulence conditions, with only a small reduction in potential core length for increased turbulence intensity.

To achieve a steady state solution required approximately 5000 timesteps using a local timestep. To achieve time-accurate unsteady solutions a global timestep was used; these calculations were much more expensive, typically requiring around 20000 time steps to achieve the unsteady flow, followed by approximately 80000 time steps on which the solution was sampled in order to compute the power spectral density.

\subsection{Steady predictions}

Initial calculations were carried out for the relatively low NPRs of 3.5 and 5.0 corresponding to the experimental work of reference [6]. Mach number contours for an NPR of 3.5 are shown in Fig. 1 to illustrate the basic features of the flow. The repeating shock cell structure can be clearly seen, resulting in the distinctive curved shear layer. To show the effect of grid resolution, Fig. 2 compares the jet centre-line Mach number for the $600 \times 210$ grid and a $300 \times 105$ grid; the increased resolution gives slightly higher peaks in the shock cell Mach number, but the shock cell wavelength and potential core length are unaffected. The methodology assumes that the total enthalpy is constant throughout the flow; to show that this is still a valid assumption, some calculations have also been carried out with the coupled solution of the transport equation for total enthalpy. Figure 3 shows the temperatures along the 


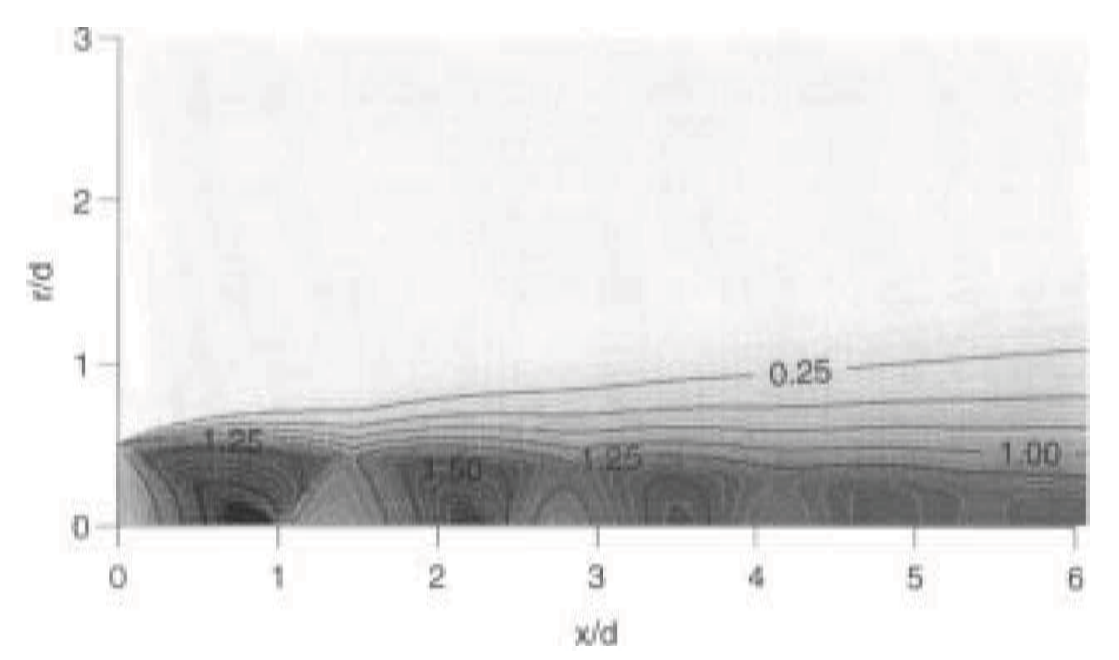

Fig. 1 Mach number contours, NPR $=3.5$

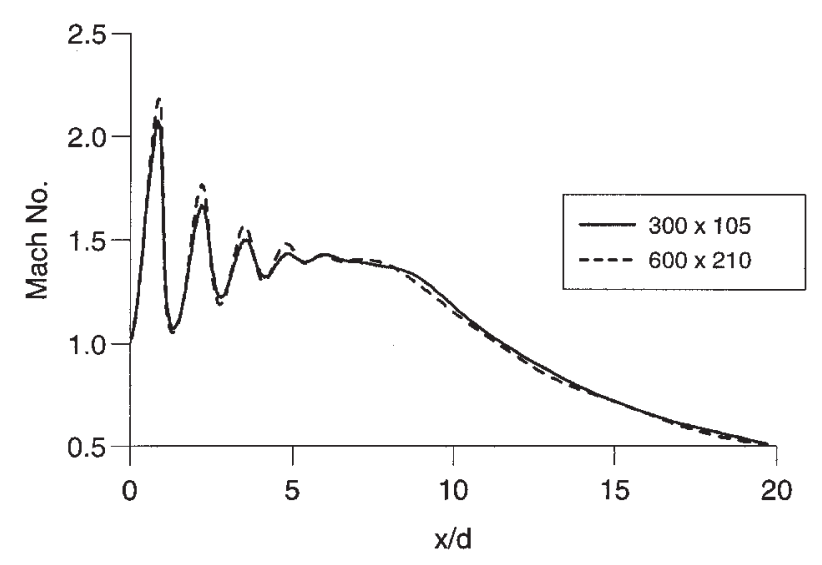

Fig. 2 Jet centre-line Mach number, grid dependence, $\mathrm{NPR}=3.5$

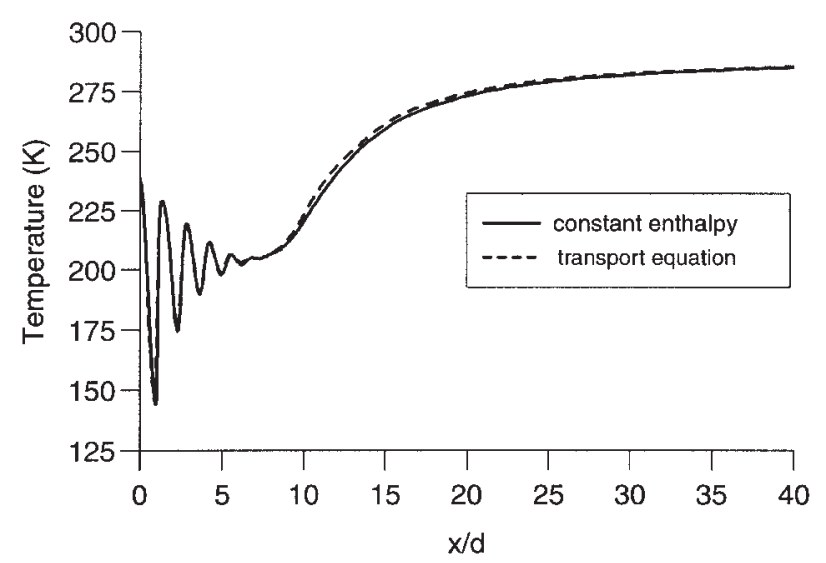

Fig. 3 Comparison of constant total enthalpy assumption with coupled transport equation for total enthalpy

jet centre-line for both calculations (with an NPR of 3.5); these are almost identical, as are all features of the flow solution.

A comparison of the jet centre-line Mach number with experiment for the lower NPR case is shown in Fig. 4 for

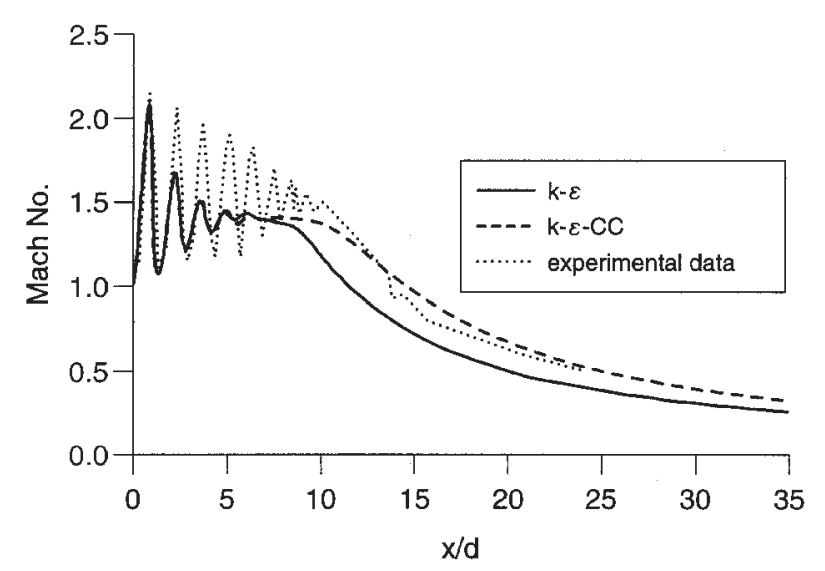

Fig. 4 Jet centre-line Mach number, effect of compressibility correction, NPR $=3.5$

both the standard and the compressibility-corrected (CC) $k-\varepsilon$ turbulence models. The shock cell wavelength is in good agreement with experiment but has decayed rather too rapidly; it is not clear whether this is due to numerical diffusion. The compressibility correction to the turbulence model has almost no effect on the shock cell structure. This is to be expected as in the jet core the magnitude of the turbulent shear stress will be small compared with the inertial terms-surprisingly, reference [12] reports that the compressibility correction is beneficial in reducing the shock cell decay. The effective reduction in turbulent viscosity in the shear layer has increased the potential core length by approximately two jet nozzle diameters, giving better agreement with the experimental potential core length than the standard model.

At higher NPRs, a normal shock or Mach disc is formed in the jet core; a typical solution showing Mach number contours is shown for an NPR of 10 in Fig. 5. The strong Mach disc is followed by a zone of subsonic flow; a slip line exists between the subsonic zone near the centre-line and the supersonic flow outside, and a triple point is 


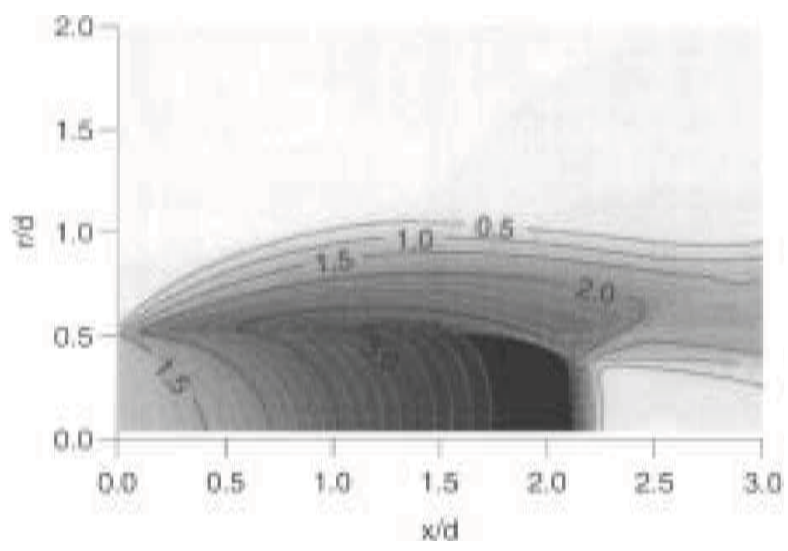

Fig. 5 Mach number contours, NPR $=10.0$

apparent at the intersection of the Mach disc with the outer flow. As will be shown in the next section, this NPR can give unsteady solutions; however, the unsteadiness is more apparent downstream of the region shown here. This is a severe test of a CFD prediction method because of the presence of a region where the local Mach number exceeds 3.5 , a strong normal shock and an outer zone where the flow is at rest and is essentially incompressible in nature.

A grid dependence test was carried out for the Mach disc cases using an NPR of 5, with jet centre-line Mach number shown in Fig. 6. The strength of the normal shock is sensitive to the axial grid resolution; the finer grid gives a sharper, stronger shock and a pronounced undershoot (normal shock equations would suggest that the downstream Mach number should be around 0.5). Downstream the two solutions are very similar. Curiously, comparison with experimental data using both the standard and the $\mathrm{CC}$ turbulence model (Fig. 7) shows the experimental data with a similar value of the Mach number aft of the shock. Downstream of the Mach disc, the mixing from the outer supersonic flow accelerates the subsonic zone and the shock cells reappear-the computations agree well with experimental data in this zone. The $\mathrm{CC}$ turbulence model

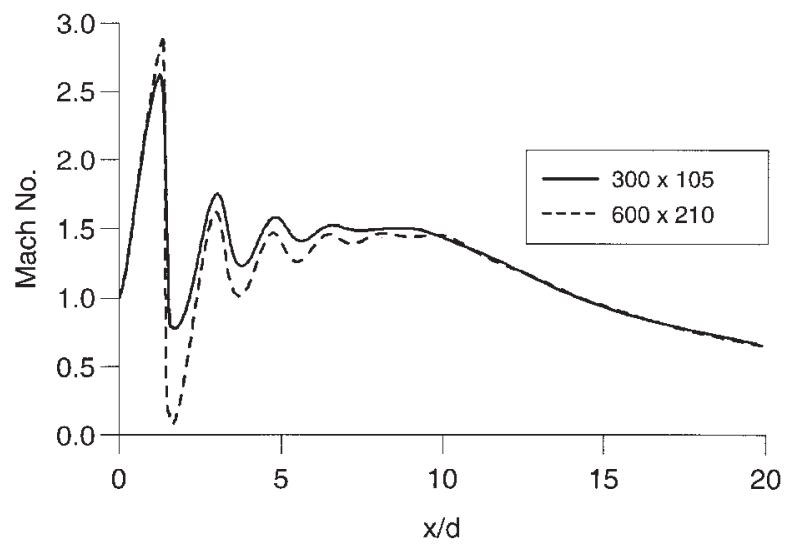

Fig. 6 Jet centre-line Mach number, grid dependence, $\mathrm{NPR}=5.0$

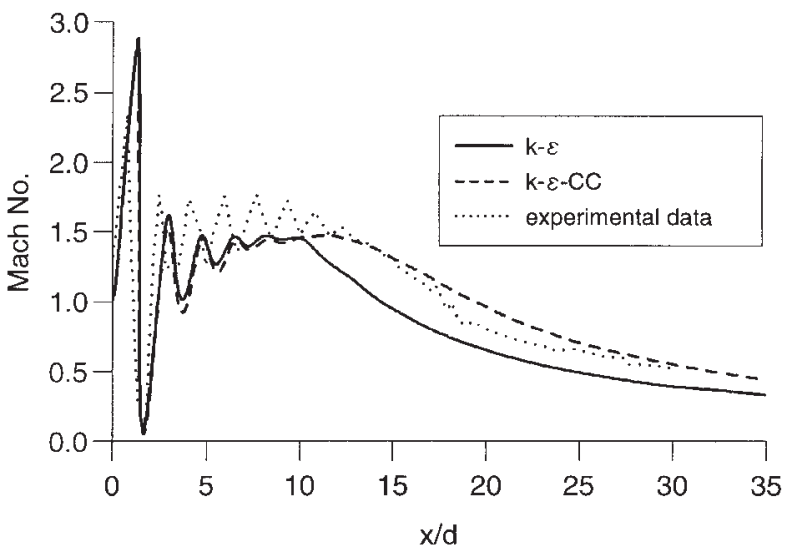

Fig. 7 Jet centre-line Mach number, effect of compressibility correction, $\mathrm{NPR}=5.0$

again gives better agreement with experimental potential core length and mixing far downstream.

A series of calculations has been carried out for highly underexpanded jets with NPRs ranging from 5 to 30. Figure 8 shows the variation of Mach disc location with NPR and is in excellent agreement with experimental data [3].

\subsection{Unsteady predictions}

For highly underexpanded jets unstable behaviour has been observed. The onset of this unstable behaviour is dependent on the pressure ratio and turbulence model. At pressure ratios above approximately 6 the jet may display these characteristics. Instabilities are more likely to be observed when the compressibility correction is used, probably because of the reduction in shear layer turbulent viscosity. Finer grids and the use of a local timestepping procedure can also accelerate the onset of unsteadiness. Tests have been carried out to remove numerical influences from the solution, such as changing timesteps, boundary locations and grid resolution. Although these factors can affect the precise nozzle pressure ratio for the onset of unsteadiness, the unstable behaviour once started is not significantly affected. An example pressure trace showing the influence

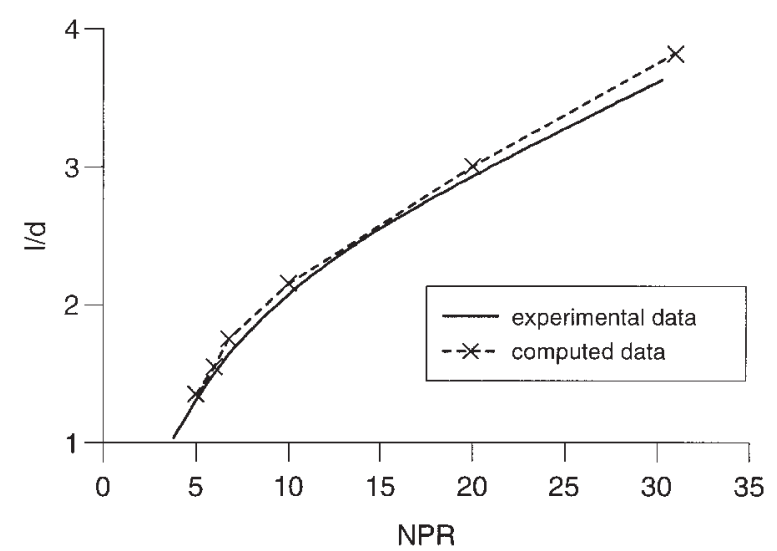

Fig. 8 Variation of Mach disc location with NPR 
of the numerical timestep is shown in Fig. 9, the smaller timestep gives a slightly larger amplitude of the oscillation, but the frequencies are identical.

While the assumption of constant total enthalpy is not strictly valid for unsteady flows, a comparison of the unsteady pressure trace using a transport equation for total energy showed the error in this assumption to be less than that due to temporal discretization error. This approximation of constant total enthalpy has been used in the unsteady calculations in order to give a useful reduction in computer time for a given calculation.

The unstable behaviour is observed in two ways. The oblique shock structure of the jet takes on a periodic structure, while large-scale structures are observed in the jet shear layers. Figures 10 and 11 show the Mach number and turbulent kinetic energy contours for an NPR of 6.0. Instabilities appear to be generated around the triple point of the Mach disc and to convect and grow in the supersonic bubble along the boundary of the potential core. These structures compress the potential core having the effect of a supersonic nozzle on the flow within it. Oblique shock

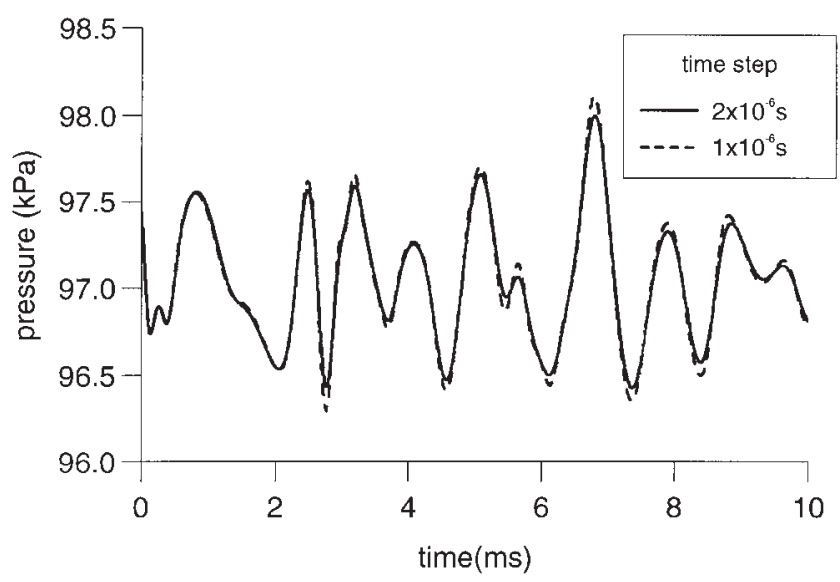

Fig. 9 Unsteady pressure trace, influence of timestep

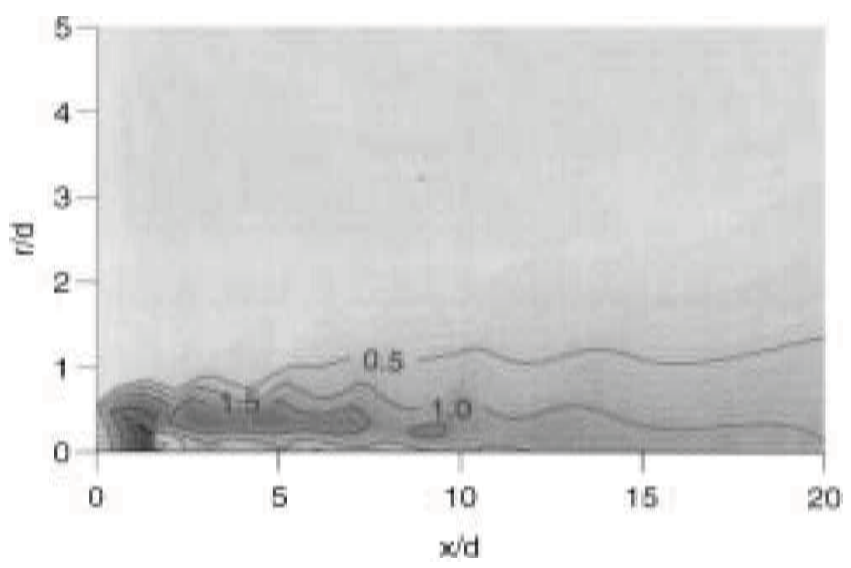

Fig. 10 Unsteady Mach number contours, NPR = 6.0

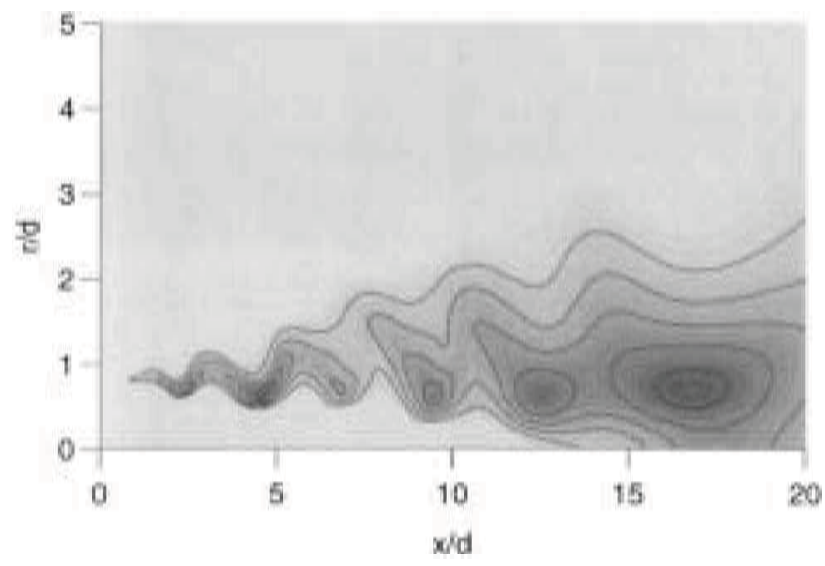

Fig. 11 Unsteady turbulence energy contours, NPR $=6.0$

waves form at these compressions, which then convect along the potential core with the large-scale structures. This gives rise to the periodic oblique shock structure that is observed in the computations. However, although the oblique shock structure is periodic, the Mach disc and the first oblique shock are stationary; instabilities only develop further downstream.

Recently there have been some experimental studies of vortex structures in high-speed jets [25-27] that indicate large scale instabilities in the shear layer. The destabilization of otherwise stable underexpanded jet flows by the presence of Kelvin-Helmholtz roll-up has been demonstrated experimentally using high speed schlieren pictures [9]. The smooth, regular shock pattern normally associated with underexpanded jets and obtained in the steady predictions was only observed in long exposure schlieren photographs, while for short exposure photographs $\left(1.1 \mu_{s}\right)$ the flow appeared to fluctuate and display a three-dimensional helical structure, which prevented identification of the shock structure downstream of the first oblique shock. This is similar to the behaviour of the unsteady predictions, although it should be noted that these are two-dimensional axisymmetric predictions and cannot resolve a threedimensional helical structure. They also showed, through an Euler flow prediction, that a time-converged solution should not be possible without suitable time averaging or by the introduction of too much dissipation. Thus, although these computations use the density-weighted ensembleaveraged Navier-Stokes equations, the time-dependent calculation appears to be reproducing some of the unsteadiness observed experimentally.

The spectral density for an NPR of 6.0 is shown in Fig. 12. This was obtained from lipline pressure-time data at $x / d=10.0$. Four modes have been predicted, with a peak mode of $4.0 \mathrm{kHz}$ and a Strouhal number of 0.16 , based on the fully expanded axial velocity. Experimental data [27] reported a dominant instability Strouhal number of around 0.18 for an NPR of 6.0, while reference [25] measured a peak frequency corresponding to a Strouhal number of 


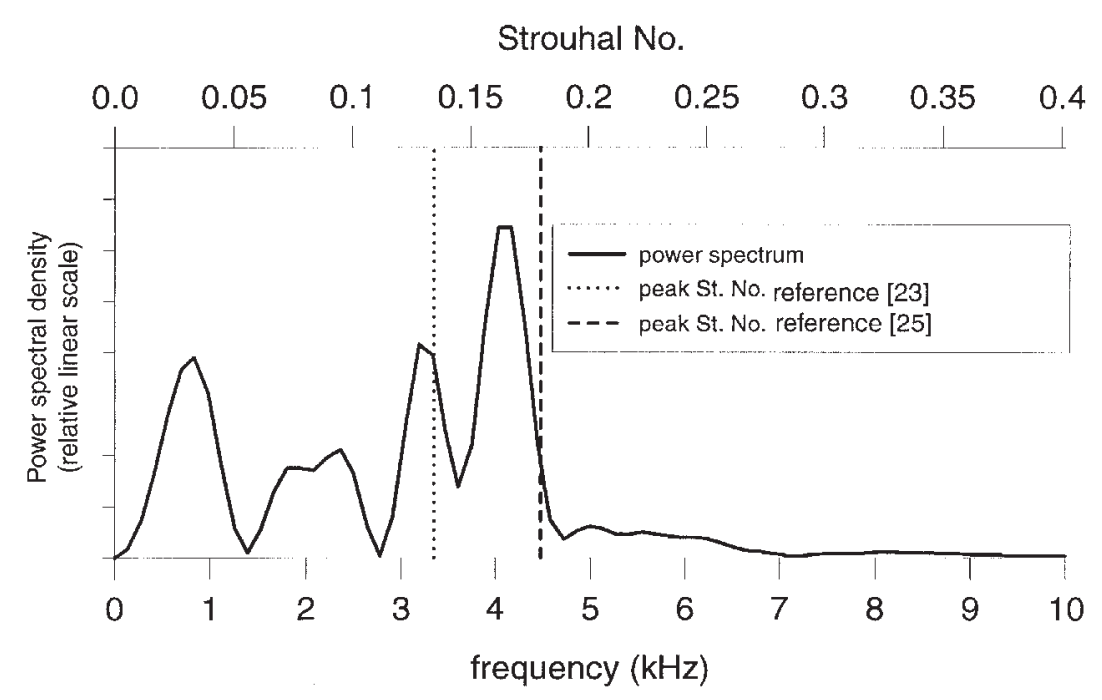

Fig. 12 Spectral analysis of unsteadiness, NPR $=6.0$

0.14. The experimental results show some disagreement, with the computed value falling between them. Experimental results for peak frequencies can be inconsistent because they are very sensitive to the experimental design such as the nozzle lip thickness and exhibit features such as hysteresis. Although it was not the original intention of this study, these calculations provide insight into some of the noise generation mechanisms in an underexpanded jet, and could be used, coupled with an appropriate method to propagate these acoustic disturbances to the far field, to predict a component of jet noise.

\section{CONCLUSIONS}

A pressure-based methodology capable of predicting underexpanded sonic jets in a quiescent domain has been applied to jet flows with NPRs from 3.5 to 30. Shock cell wavelength is correctly predicted, but comparison with experiment indicates a too rapid decay; this may be due to numerical diffusion related to the large streamwise gradients. The compressibility correction to the turbulence model has no effect on the shock cell decay but increases the potential core length to give better agreement with experiment than the standard model. For highly underexpanded jets, the Mach disc location is correctly predicted and the distinctive physical features such as the triple point, slip line and the curved jet boundary have been resolved.

Unsteady solutions have been predicted, corresponding to unsteadiness observed in experimental work. The power spectral density computed from pressure data at the nozzle exit contains a peak equivalent to a Strouhal number of 0.16; this compares favourably with experiment. The predicted unsteadiness could potentially be used, coupled to an acoustic model, to predict a component of the farfield jet noise.

\section{ACKNOWLEDGEMENTS}

The authors would like to thank the Engineering and Physical Sciences Research Council of the United Kingdom for the support of this work and our colleagues Professor J. C. Dent, Dr W. M. Malalasekera and Professor J. J. McGuirk for their useful discussions.

\section{REFERENCES}

1 Pack, D. C. On the formation of shock-waves in supersonic gas jets. Q. J. Mechanics Appl. Math., 1948, 1, 1-17.

2 Adamson Jr, T. C. and Nicholls, J. A. On the structure of jets from highly underexpanded nozzles into still air. J. Aerospace Sci., 1959, 26, 16-24.

3 Love, E. S., Grigsby, C. E., Lee, L. P. and Woodling, J. M. Experimental and theoretical studies of axisymmetric free jets. NASA Technical Report R-6, 1959.

4 Crist, S., Sherman, P. M. and Glass, D. R. Study of highly underexpanded sonic jet. Am. Inst. Aeronaut. Astronaut. J., 1966, 4, 68-71.

5 Donaldson, C. D. and Snedeker, R. S. A study of free jet impingement. Part 1. Mean properties of free and impinging jets. J. Fluid Mechanics, 1971, 45, 281-319.

6 Stickland, M. T., Green, L. G. and Russell, C. RA98 (CHAM Nozzle) flow survey of underexpanded supersonic jets in the $5.5 \mathrm{~m}$ low speed wind tunnel static test facilityvolume 2. British Aerospace Report BAe-WWt-RP-RESAXR-139, 1988.

7 Birch, A. D., Hughes, D. J. and Swaffield, F. Velocity decay 
of high pressure jets. Combust. Sci. Technol., 1987, 52, 161-171.

8 Chuech, S. G., Lai, M.-C. and Faeth, G. M. Structure of turbulent underexpanded free jets. Am. Inst. Aeronaut. Astronaut. J., 1989, 27, 549-559.

9 Matsuda, T., Umeda, Y., Ishii, R., Yasuda, A. and Sawada, K. Numerical and experimental studies on choked underexpanded jets. AIAA paper 87-1378, 1987.

10 Dash, S. M., Wolf, D. E. and Seiner, J. M. Analysis of turbulent underexpanded jets, Part I: parabolized NavierStokes model, SCIPVIS. Am. Inst. Aeronaut. Astronaut. J., 1985, 23, 505-514.

11 Abdol-Hamid, K. S. and Wilmoth, R. G. Multiscale turbulence effects in underexpanded supersonic jets. Am. Inst. Aeronaut. Astronaut. J., 1989, 27, 315-322.

12 Cumber, P. S., Fairweather, M., Falle, S. A. E. G. and Giddings, J. R. Predictions of the structure of turbulent, moderately underexpanded jets. Trans. ASME, J. Fluids Engng, 1994, 116, 703-713.

13 Palacio, A., Malin, M. R., Proumen, N. and Sanchez, L. Numerical computation of steady transonic and supersonic flow fields. Int. J. Heat Mass Transfer, 1990, 33, 1193-1204.

14 Cumber, P. S., Fairweather, M., Falle, S. A. E. G. and Giddings, J. R. Predictions of the structure of turbulent, highly underexpanded jets. Trans. ASME, J. Fluids Engng, 1995, 117, 599-604.

15 Papamoschou, D. and Roshko, A. The compressible turbulent shear layer: an experimental study. J. Fluid Mechanics, 1988, 197, 453-477.

16 Zeman, O. Dilatation dissipation: the concept and application in modelling compressible mixing layers. Physics Fluids A (Fluid Dynamics), 1990, 2, 176-188.
17 Sarkar, S., Erlebacher, G. and Hussaini, M. Y. The analysis and modelling of dilatational terms in compressible turbulence. J. Fluid Mechanics, 1991, 227, 473-493.

18 Speziale, C. G. and Sarkar, S. Second-order closure models for supersonic turbulent flows. AIAA paper 91-0217, 1991.

19 Sarkar, S. The stabilizing effect of compressibility in turbulent shear flow. J. Fluid Mechanics, 1995, 282, 163-186.

20 Vreman, A. W., Sandham, N. D. and Luo, K. H. Compressible mixing layer growth rate and turbulence characteristics. J. Fluid Mechanics, 1996, 320, 235-258.

21 Jones, W. P. and Launder, B. E. The prediction of laminarisation with a two-equation model of turbulence. Int. J. Heat Mass Transfer, 1972, 15, 301-314.

22 McGuirk, J. J. and Page, G. J. Shock capturing using a pressure-correction method. Am. Inst. Aeronaut. Astronaut. J., 28, 1990, 1751-1757.

23 McGuirk, J. J. and Page, G. J. The calculation of underexpanded impinging jets. In Fourth Symposium on Numerical and Physical Aspects of Aerodynamic Flows, Long Beach, California, 16-19 January 1989.

24 Demirdzic, I., Lilek, Z. and Peric, M. A collocated finite volume method for predicting flows at all speeds. Int. J. Numer. Meth. Fluids, 1993, 16, 1029-1050.

25 Powell, A., Umeda, Y. and Ishii, R. Observations of the oscillation modes of choked circular jets. J. Acoust. Soc. Am., 1992, 92, 2823-2836.

26 Gutmark, E., Schadow, K. C. and Bicker, C. J. Mode switching in supersonic circular jets. Physics Fluids A (Fluid Dynamics), 1989, 1, 868-873.

$27 \mathrm{Hu}$, T. F. and McLaughlin, D. K. Flow and acoustic properties of low Reynolds-number underexpanded supersonic jets. J. Sound Vibr., 1990, 141, 485-505. 